\title{
La dependencia importadora de la economía española
}

\author{
ROSARIO GANDOY \\ Universidad de Castilla La Mancha, Facultad de Ciencias Jurídicas y Sociales, Cobertizo de San \\ Pedro mártir s/n, 45071 Toledo, España.E-mail: Rosario.gandoy@uclm.es
}

\begin{abstract}
RESUMEN
Las estimaciones efectuadas a partir de las Tablas Input-Output Internacionales revelan que la dependencia importadora de la economía española, tras quebrar su tendencia creciente en 2009, recuperó rápidamente sus niveles previos a la crisis. Aunque en el conjunto europeo no parece especialmente elevada, supera a la de las economías centrales europeas, en especial, en las manufacturas de mayor intensidad tecnológica. Ello parece ser el resultado de una especialización productiva de carácter vertical, vinculada al capital extranjero, en la que las empresas españolas abordan las etapas de transformación y montaje, pero también del impulso a estrategias de abastecimiento externo con el objetivo de incrementar la productividad y competitividad. Adicionalmente, la elevada dependencia relativa de importaciones finales de alta tecnología refleja el insuficiente desarrollo de estas producciones en nuestro país.

Palabras clave: Dependencia importadora, análisis input-output, comercio exterior.
\end{abstract}

\section{The Import Dependence of Spanish Economy}

\begin{abstract}
Using International Country Input-Output Tables this paper provides empirical evidence on the evolution of import content of the different components of global demand in Spain. Import content registered a significant increase since 1995 until trade collapsed in 2009; after the fall it recovered its pre-crisis levels in 2011. Spanish import penetration is lower than most European countries are, but higher than that of the four largest EU countries, particularly in high-tech industries. This higher import content might be explained by firm's involvement in global value chains controlled by multinational companies as well as by firm's engagement in international sourcing strategies to improve productivity and competitiveness. Finally, the deep dependence of final imports for high-tech industries reflects a lack of production capacity in those industries due to domestic technological weaknesses.
\end{abstract}

Keywords: Import content, Input-Output Analysis, International Trade.

Clasificación JEL: F10, F14

Artículo recibido en octubre de 2016 y aceptado en noviembre de 2016

Artículo disponible en versión electrónica en la página www.revista-eea.net, ref. ə-35105 


\section{INTRODUCCIÓN}

Las importaciones españolas, que mostraron un elevado dinamismo desde mediados de los noventa hasta el comienzo de la crisis financiera internacional, sufrieron una intensa contracción en 2009, iniciándose a partir de entonces un proceso de ajuste que se extiende hasta los primeros indicios de recuperación en 2013. Este comportamiento de las importaciones, acompañado de la expansión de las exportaciones, posibilitó durante la fase recesiva del ciclo la corrección del considerable desequilibrio exterior que había alcanzado nuestra economía en los años previos a la crisis (cerca del 10\% del PIB en 2007), pasando a registrarse saldos positivos desde el año 2012.

Sin embargo, la incipiente recuperación de la demanda en 2014 y 2015 se ha traducido en un progresivo aumento de las compras al exterior que ha generado inquietud acerca de cuáles son los factores que determinan el comportamiento importador y, en consecuencia, sobre la capacidad de la economía española para mantener el equilibrio con el exterior en un contexto de crecimiento de la demanda. Si el ajuste en las importaciones se debe a factores cíclicos, es decir, si es la respuesta ante la profundidad de la caída en la demanda interna, es previsible un comportamiento expansivo de las compras exteriores ante la reactivación de la demanda. En cambio, si son factores estructurales los que han propiciado el ajuste (v.g. una sustitución de importaciones por producción nacional derivada de la mejora en la competitividad asociada al proceso de devaluación interna o al cambio en el modelo productivo) sería de esperar una menor elasticidad de las importaciones respecto a la demanda final.

El debate sobre el carácter cíclico o estructural de los flujos comerciales no es específico de la economía española. El colapso del comercio mundial en 2009 y su posterior desaceleración han generado una abundante literatura que trata de determinar si la menor contribución del comercio al crecimiento económico es de carácter coyuntural o perdurable en el tiempo ${ }^{1}$. Sin embargo, los resultados obtenidos no son concluyentes. Mientras que algunos de los trabajos encuentran que la senda reciente de los flujos comerciales se explica fundamentalmente por la evolución y cambios en la composición de la demanda (Bussière et al., 2013; BCE, 2015), otros justifican su lento ritmo de avance por factores estructurales, entre los que destaca la incidencia de las cadenas globales de valor (Escaith, 2010; Constantinescu et al., 2015).

El presente trabajo, con un planteamiento más modesto, trata de contribuir a explicar el comportamiento de las importaciones españolas analizando la dependencia exterior de la demanda teniendo en cuenta sus distintos componentes (consumo privado, público, inversión y exportaciones). La dependencia del exterior se manifiesta tanto en las compras de bienes finales al resto del mundo

\footnotetext{
${ }^{1}$ Hoekman (2015) recoge un amplio conjunto de aportaciones al respecto.
} 
para satisfacer la demanda nacional como en el contenido importado de las producciones españolas destinadas al consumo, inversión o exportaciones, es decir, por los requerimientos unitarios de inputs intermedios procedentes del exterior (directos e indirectos) que tiene el tejido productivo. Permite aproximar, por tanto, la elasticidad de las importaciones respecto a la demanda final.

Tradicionalmente, la dependencia importadora de la demanda se ha estimado a partir de las tablas input-output nacionales (v.g. Cabrero y Tiana, 2012). La novedad de este trabajo es su medición a partir de las tablas input-output internacionales, que posibilitan una estimación más afinada. En primer lugar, porque permiten distinguir el origen geográfico de las importaciones por ramas de actividad destinadas a usos finales e intermedios y posibilita conocer la dependencia importadora por países, sectores y componentes de la demanda. En segundo lugar, porque al ser una tabla global que integra de forma armonizada las relaciones interindustriales y el equilibrio entre la oferta y la demanda de distintas economías, facilita la comparación entre países, pudiéndose detectar características y evoluciones específicas. Y, en tercer lugar, porque permite descomponer el valor añadido incorporado en las importaciones según su procedencia nacional o extranjera.

Este último punto es especialmente relevante para el análisis, puesto que constituye una clara mejora frente a estimaciones previas. Las tablas internacionales surgen como respuesta a los cambios en el comercio internacional provocados por la globalización económica y la internacionalización de la producción. En un marco de intensa fragmentación internacional de los procesos productivos, las estadísticas de comercio tradicionales pierden su capacidad para valorar adecuadamente las relaciones comerciales entre los países. Cuando los procesos de fabricación se encuentran dispersos geográficamente y los inputs intermedios cruzan sucesivas fronteras para irse transformando hasta su incorporación en el bien final (quizás exportado), se produce una múltiple contabilización de los inputs comerciados, de modo que el valor de las exportaciones finales no se corresponde con el valor añadido; con las rentas que se generan en el país. Las tablas internacionales permiten identificar el valor añadido que cada país aporta a las cadenas globales de valor a través de la descomposición del valor añadido incorporado en los flujos comerciales.

En lo que respecta a nuestro objetivo, la dependencia exterior de la economía, hay que considerar que parte de las importaciones finales pueden incorporar inputs intermedios generados en el propio país que se exportaron para su transformación en el país que ahora suministra el bien final. Sería el caso, por ejemplo, de la fabricación y exportación de tela a Marruecos para la confección de trajes y la posterior importación de estos trajes para satisfacer la demanda de consumo interior. Al objeto de valorar adecuadamente el contenido foráneo de la demanda final, el valor de los inputs nacionales importados (la tela con que se 
confeccionan los trajes adquiridos a Marruecos) ha de ser excluido, lo que no ha sido posible hasta la aparición de las tablas input-output internacionales.

No obstante, las tablas input-output internacionales siguen mostrando algunas de las limitaciones propias de este instrumento de análisis que obligan a valorar con cautela los resultados obtenidos. Así, la valoración a precios corrientes de las magnitudes puede condicionar la evolución de los indicadores si la evolución de los precios sectoriales es muy dispar y se producen alteraciones fuertes en los tipos de cambio de las monedas. Entre los supuestos que incorporan se considera que todos los productos englobados en un determinado sector se generan de acuerdo a la misma estructura técnica, con las mismas necesidades de inputs intermedios y que las empresas de un sector son homogéneas en sus procesos productivos. Dado que es bien conocido que las empresas exportadoras tienen características superiores en cuanto a su eficiencia productiva y grado de internacionalización, este supuesto impide distinguir con propiedad la dependencia importadora de los bienes y servicios destinados a los mercados exteriores del resto, de modo que las diferencias en el contenido importado de las exportaciones ha de atribuirse a la disparidad entre el patrón comercial y productivo ${ }^{2}$.

El resto del trabajo se estructura de la siguiente manera. En el siguiente apartado se explicita la estructura de las tablas input-output internacionales y la metodología empleada para estimar la dependencia importadora. Los resultados de esta estimación para la economía española y su comparación con otras economías avanzadas se presentan en el epígrafe tres. A continuación, se lleva a cabo el análisis sectorial $\mathrm{y}$, tras algunas consideraciones respecto a las implicaciones de la dependencia importadora, el artículo se cierra con unas conclusiones.

\section{EL VALOR AÑADIDO INCORPORADO EN LA DEMANDA FINAL}

Las Tablas input-output internacionales proporcionan una visión integrada de la estructura productiva y las relaciones comerciales de las economías que se incluyen en ellas. Su aparición está vinculada a la expansión internacional de los procesos productivos y su objetivo principal es distinguir la participación en los flujos comerciales del valor añadido generado en el propio país y en el exterior. Aunque en la actualidad se disponen de varias tablas globales, en ese trabajo se utilizan las International Country Input Output (ICIO), elaboradas por la OCDE y la Organización Mundial del Comercio ${ }^{3}$. Esta fuente estadística

\footnotetext{
${ }^{2}$ Salvo para China y Méjico, para los que las tablas input-output internacionales de la OCDE distinguen los intercambios intersectoriales en la producción vinculada al comercio de procesamiento del resto de transacciones.

3 Además de la ICIO están disponibles las siguientes tablas input-output internacionales: la UNCTAD-Eora que considera un conjunto más amplio de países, especialmente, de los países
} 
se basa en las tablas input-output oficiales de los distintos países que las conforman, que son integradas a través de los flujos de comercio bilateral que se obtienen a partir de la base de datos COMTRADE que elabora Naciones Unidas. La tabla global proporciona información acerca de las interrelaciones que tienen lugar entre las actividades productivas de una economía, entre estas y las diferentes producciones del resto de países, y sus relaciones con los componentes de la demanda final nacional y foránea. En particular, las ICIO consideran 34 sectores de actividad y 61 países, más un agregado para el resto del mundo. La información está disponible para los años 1995, 2000, 2005 y anualmente desde 2008 a 2011.

La estructura de las tablas internacionales, que sigue el esquema habitual de las tablas input output nacionales, se refleja en la Figura 1. La matriz de relaciones interindustriales consta de $n^{*} k$ filas y $n^{*} k$ columnas (siendo $n$ el número de ramas de actividad y $k$ el de países) y recoge los intercambios de inputs intermedios entre sectores y países, de tal manera que el elemento $X_{i j}{ }^{c p}$, muestra las compras que la rama $j$ del país $p$ (sector-país columna) adquiere de la rama $i$ procedente del país $c$ (sector-país fila). La suma de las columnas muestra el total de consumos intermedios que, de acuerdo con la tecnología y estructura productiva, se requiere para llevar a cabo la producción de la ramapaís columna. Nótese que la ICIO permite discernir las compras intermedias de bienes nacionales (cuando $c=p$ ) de las importaciones intermedias (cuando $c \neq p$ ).

Figura1

Esquema de la tabla input-output internacional

\begin{tabular}{|c|c|c|c|c|c|c|c|c|c|c|c|}
\hline & & & \multicolumn{4}{|c|}{ Empleo por pais-sector } & \multicolumn{4}{|c|}{ Demanda final } & \multirow{3}{*}{$\begin{array}{c}\text { Empleos } \\
\text { totales }\end{array}$} \\
\hline & & & \multicolumn{2}{|c|}{ Pais 1} & \multicolumn{2}{|c|}{ Pais $\mathrm{K}$} & \multicolumn{2}{|c|}{ Pais 1} & \multicolumn{2}{|c|}{ Pais $\mathrm{K}$} & \\
\hline & & & Sector 1 & Sector $n$ & Sector 1 & Sector $\mathrm{n}$ & DF 1 & $D F f$ & DF 1 & DF $f$ & \\
\hline \multirow{7}{*}{$\begin{array}{l}\text { Origen inputs: pais- } \\
\text { sector }\end{array}$} & \multirow{3}{*}{ Pais 1} & Sector 1 & & & & & & & & & \\
\hline & & $\ldots$ & & & & & & & & & \\
\hline & & Sector $\mathrm{n}$ & & & & & & & & & \\
\hline & $\ldots$ & & & & & & & & & & \\
\hline & \multirow{3}{*}{ Pais K } & Sector 1 & & & & & & & & & \\
\hline & & $\ldots$ & & & & & & & & & \\
\hline & & Sector $\mathrm{n}$ & & & & & & & & & \\
\hline \multicolumn{3}{|c|}{ Valor añadido por trabajo y capital } & & & & & & & & & \\
\hline Output & & & & & & & & & & & \\
\hline
\end{tabular}

Fuente: Adaptado de Timmer et al. (2015).

La matriz de demanda final, colocada a la derecha de la matriz de inputs intermedios, está formada por $n^{*} k$ filas y $k^{*} f$ columnas, siendo $f$ los componentes

menos desarrollados; la WIOD impulsada por la Comisión Europea, la GTAP que elabora la Universidad de Purdue y las tablas input-output internacionales asiáticas (IDE-JETRO). Jones et al. (2014) comparan la información que proporcionan y los indicadores de comercio en valor añadido que se deprenden de las tablas ICIO, WIOD y GTAP. 
de la demanda final (consumo privado, consumo público, inversión). Cada uno de sus elementos, $d_{i}^{c p}$, expresa la demanda final que realiza el país columna, $p$, de bienes y servicios del sector $i$ procedentes del país fila, $c$. De nuevo, es posible diferenciar la parte de cada componente de la demanda final que se cubre con producción doméstica $(c=p)$ de la que se satisface con importaciones finales $(c \neq p)$.

Finalmente, las tablas incorporan, al final de la matriz de relaciones interindustriales, un vector fila de valor añadido de orden $n * k$, que registra el valor añadido (rentas) generado en cada rama por el sector columna. La suma de cada columna de la matriz de relaciones interindustriales y su correspondiente valor añadido es el valor de producción de cada rama productiva en cada país. Su cálculo para todos los países y sectores permite obtener un vector fila de output de orden $n * k$.

La estimación de la dependencia externa requiere conocer cuál es el valor añadido procedente del exterior incorporado en la demanda final de una economía, tanto directamente a través de importaciones de bienes finales, como indirectamente mediante la adquisición de los inputs importados que se precisan para llevar a cabo la producción nacional. La metodología empleada parte del conocido modelo de demanda de Leontief $^{4}$ y de la descomposición del valor añadido incorporado en las exportaciones propuesta por Koopman et al., 2014 ${ }^{5}$.

El valor añadido incorporado en la demanda final puede calcularse como

$$
V A D=\widehat{V}[I-A]^{-1} \widehat{D}
$$

Donde

$\widehat{V}$ es una matriz diagonal de orden $n * k$ (siendo $n=34$ y $k=62$ ) cuyos elementos de la diagonal principal son los coeficientes de valor añadido, $v_{j}^{p}$, que reflejan el porcentaje que supone el valor añadido generado en la rama $j$ sobre el total de la producción de $j$ en el país $p$.

$[I-A]^{-1}$ es la matriz inversa de Leontief. Es una matriz de orden $n^{*} k$ que registra las necesidades directas e indirectas de inputs intermedios por unidad de producto. Cada elemento de la matriz, $b_{i j}^{c p}$, muestra las necesidades de

\footnotetext{
${ }^{4}$ Una descripción sencilla del modelo de demanda de Leontief puede encontrarse en Muñoz (2000).

${ }^{5}$ La distribución del valor añadido de las exportaciones estimada a partir de las tablas input-output internacionales ha posibilitado el desarrollo de recientes investigaciones dirigidas principalmente a analizar la entidad y naturaleza de las cadenas globales de valor (v.g. Baldwin y López-González, 2013; Amador et al., 2015; De Backer y Miroudot, 2013; Timmer et al., 2014) y a examinar las implicaciones de la participación en cadenas globales de valor en la política económica (v.g. Kowalski et al., 2015 y Escaith, 2014).
} 
inputs intermedios de la rama $i$ procedentes del país $c$ que se requieren en el país $p$ para atender a una unidad de la demanda final del bien $j$.

$\widehat{D}$ es una matriz diagonal de demanda final, cuya diagonal toma distintos valores según cuál sea el componente de la demanda cuya dependencia importadora se desee estudiar (total, consumo privado, consumo público, inversión ${ }^{6}$ o exportaciones). Cada elemento, $d_{i}^{c p}$, muestra la demanda final del bien $i$ que hace el país $p$ y se satisface con producciones procedentes del país $c$.

$V A D$ es la matriz de orden $n * k$ resultante. Cada elemento, $v a d_{i j}^{c p}$, recoge el valor añadido de la rama i procedente del país c que se incorpora en el bien $\mathrm{j}$ procedente del país $p$ para satisfacer la demanda final (total, consumo privado, público, inversión o exportaciones) de un determinado país.

La demanda final un bien o servicio puede satisfacerse con producción nacional (demanda final doméstica) o importaciones finales.

Las columnas de VAD correspondientes al país cuya demanda final se está estudiando (una para cada bien) recogen el valor añadido incorporado en la demanda final doméstica, es decir, aquella que es satisfecha con producción generada en el propio país. En ellas se puede distinguir las rentas que en el proceso de producción se generan en el propio país de las que proceden del exterior. Formalmente, y de forma genérica, cuando $p$ el país cuya demanda final se está analizando, el valor añadido contenido en la demanda doméstica que el país $p$ hace del bien $i$ será:

$$
\text { VADemanda final doméstica } i^{p}=\sum_{j} v a d_{j i}^{p p}+\sum_{j, c \neq p} v a_{j i}^{c p}
$$

siendo el primer término las rentas nacionales y el segundo las que se generan en el exterior y se incorporan en la producción nacional de $i$ a través de importaciones intermedias que constituyen el contenido importado.

Las columnas correspondientes al resto de países reflejan el valor añadido incorporado en las importaciones finales destinadas a satisfacer la demanda final de $p$. Formalmente, la descomposición del valor añadido incorporado en las importaciones finales, cuando se amplía el número de países y sectores puede expresarse como:

$$
\text { VAImportaciones finales } i^{p}=\sum_{j, c \neq p} v_{j i}^{c c}+\sum_{j, c \neq p} \operatorname{vad}_{j i}^{p c}
$$

El primer término de la igualdad refleja el valor añadido exterior incluido en

\footnotetext{
${ }^{6}$ La información que proporcionan las ICIO sobre componentes de la demanda final incluye la Formación Bruta de Capital Fijo y Variación de Existencias. Sin embargo, dada la variabilidad de estas últimas y tal como sugieren Bussière et al. (2013) y OCDE (2015b), para estimar la intensidad importadora en la inversión se ha considerado exclusivamente la Formación Bruta de Capital Fijo.
} 
las importaciones finales del bien $i$. El segundo, es el valor añadido generado en el país $p$ que se incorpora en las importaciones finales que $p$ efectúa.

En definitiva, el valor añadido del exterior que se incorpora en la demanda final de un bien puede definirse como:

$$
\text { VA foráneoincorporado en la demanda finali }{ }^{p}=\sum_{j, c \neq p} v a d_{j i}^{c c}+\sum_{j, c \neq p} v_{j i}^{c p}
$$

Su cociente respecto a la demanda final mide la dependencia importadora de la rama $i$. Agregando el valor añadido foráneo para todas las ramas de actividad se obtiene la dependencia importadora de los distintos componentes de la demanda final de la economía, distinguiendo entre dependencia directa, la relativa a las importaciones finales destinadas a satisfacer la demanda e indirecta que recoge el contenido importado en la producción nacional que cubre la demanda final (consumo público, privado, inversión y exportaciones).

\section{LA DEPENDENCIA EXTERIOR DE LA DEMANDA}

La Tabla 1 recoge los resultados de la estimación realizada para la economía española ${ }^{7}$. Los valores muestran el porcentaje que supone el valor añadido foráneo sobre la demanda final de cada uno de los componentes de la demanda. La última fila del cuadro recoge la dependencia del exterior de la economía, incorporando la de la demanda final nacional y exterior (exportaciones). En este caso el ratio representa su porcentaje sobre la demanda total (nacional y exterior).

El primer rasgo a destacar es el fuerte aumento de la dependencia que ha tenido lugar en la economía española desde mediados de los años noventa, especialmente en la segunda mitad de dicha década cuando la contribución del resto del mundo a la oferta total de bienes y servicios aumentó un 35\% hasta situarse en el 25,2\% en el año 2000. Desde entonces, este porcentaje se ha mantenido estable hasta el colapso del comercio mundial en 2009, cuando se redujo sustancialmente. Si bien, ya en 2011, mucho antes de que la recuperación económica empezase a manifestarse en nuestro país, se observa un retorno a los valores previos a la crisis.

La mayor dependencia externa se registra en las exportaciones, con una presencia externa en la producción que alcanza el 26,8\% en 2011 y supera

\footnotetext{
${ }^{7} \mathrm{Al}$ igual que en la mayor parte de los trabajos que analizan la dependencia importadora no se considera la reexportación de bienes finales, es decir, la exportación de productos finales importados. Como señalan Bussière et al. (2013), en las economías avanzadas la importancia que alcanzan las importaciones destinadas a la reexportación es limitada, de modo que los resultados no se ven significativamente afectados por este supuesto. Asimismo, para la estimación de la dependencia importadora del consumo privado no se ha considerado el consumo de los residentes en el exterior.
} 
durante todo el periodo la del conjunto de la demanda final nacional (23,7\% en 2011).

El contenido importado de las exportaciones, ha sido habitualmente empleado como indicador de la especialización vertical (Hummels et al., 2001) y, más recientemente, de la participación en cadenas globales de valor a través de la transformación de bienes intermedios para su venta en los mercados exteriores. Así, la elevada y creciente penetración de importaciones en los bienes exportados que muestra la Tabla 1, especialmente intensa en el primer quinquenio estudiado, puede interpretarse como reflejo de la progresiva inserción de la economía española en procesos de fragmentación internacional de la producción. La internacionalización de los procesos productivos experimentó un considerable impulso desde mediados de los años noventa, favoreciendo el comercio mundial de bienes intermedios, que creció a tasas superiores a las del resto de bienes conforme se llevaba a cabo la extensión geográfica de las cadenas de producción. Nuestro país no ha sido ajeno a esta tendencia y en línea el resto de las economías, tanto avanzadas como emergentes, incrementó su grado de especialización vertical (OCDE, 2011; Breda, 2009). El análisis sectorial que se efectúa en el siguiente apartado, permitirá ahondar en esta cuestión.

Tabla 1

Dependencia exterior de la demanda por componentes (Porcentajes)

\begin{tabular}{|cccccc|}
\hline & $\mathbf{1 9 9 5}$ & $\mathbf{2 0 0 0}$ & $\mathbf{2 0 0 8}$ & $\mathbf{2 0 0 9}$ & $\mathbf{2 0 1 1}$ \\
\hline DEPENDENCIA INDIRECTA: CONTENIDO IMPORTADO & & & & \\
\hline Demanda final nacional & 10,6 & 13,3 & 13,5 & 10,9 & 12,5 \\
Consumo privado & 11,0 & 13,6 & 14,0 & 11,6 & 13,3 \\
Consumo público & 6,3 & 8,4 & 9,2 & 8,1 & 9,2 \\
Formación Bruta de Capital & 13,8 & 16,4 & 16,1 & 12,5 & 14,7 \\
Exportaciones & 19,1 & 25,7 & 27,4 & 22,1 & 26,8 \\
\hline DEPENDENCIA DIRECTA: IMPORTACIONES FINALES & & & & \\
\hline Demanda final nacional & 7,9 & 11,7 & 10,6 & 9,3 & 11,1 \\
Consumo privado & 9,2 & 12,8 & 12,8 & 12,0 & 13,6 \\
Consumo público & 0,6 & 0,9 & 1,1 & 0,9 & 1,0 \\
Formación Bruta de Capital & 10,6 & 16,4 & 13,0 & 10,8 & 15,1 \\
\hline DEPENDENCIA IMPORTADORA & & & & & 23,7 \\
\hline Demanda final nacional & 18,6 & 25,0 & 24,1 & 20,3 & 26,9 \\
Consumo privado & 20,2 & 26,5 & 26,8 & 23,6 & 10,2 \\
Consumo público & 6,9 & 9,3 & 10,4 & 9,0 & 29,8 \\
Formación Bruta de Capital & 24,5 & 32,9 & 29,0 & 23,3 & $\mathbf{2 4 , 5}$ \\
\hline
\end{tabular}

Fuente: Elaboración propia a partir de ICIO.

La notable recuperación del contenido importado en las exportaciones que se observa en 2011, y que también tiene lugar en la mayor parte de las economías, sugiere que la caída que se produjo en 2009 no se debió tanto a un cambio en la 
tendencia a la internacionalización de la producción como a una interrupción provocada por la superior incidencia de la crisis sobre algunas de las ramas de actividad más dependientes de inputs importados.

Tras las exportaciones, el componente de la demanda final que muestra un mayor grado de dependencia externa es la inversión. Tanto sus requerimientos unitarios de importaciones intermedias como de bienes finales superan a los del resto de componentes de la demanda nacional; salvo en 2009, cuando la sensibilidad de las importaciones de bienes de inversión ante la contracción de la demanda fue mayor que en el consumo. No obstante, el rebrote en las importaciones finales en 2011, cuando cubrieron un $15 \%$ de la demanda de inversión, un porcentaje superior al vigente en los años previos a la crisis, descarta la posibilidad de un proceso de sustitución de importaciones en los bienes de inversión.

La elevada dependencia importadora de la demanda de inversión podría vincularse con la limitada capacidad investigadora de la economía española y su reducido esfuerzo en investigación y desarrollo, que determina la necesidad de que las empresas adquieran del exterior sus bienes de equipo de mayores exigencias tecnológicas. No obstante, como muestra el trabajo de Bessière et al. (2013), las exportaciones y demanda de inversión son los elementos de la demanda que muestran un mayor contenido importado en la mayor parte de las economías.

De hecho, de los 18 países de la OCDE que estos autores consideran en su estimación para 2005 a partir de las tablas input-output nacionales, en todos excepto el Reino Unido, la dependencia importadora de la inversión supera a la del consumo privado. Por tanto, la elevada dependencia importadora de la demanda de inversión constituye un hecho estilizado. El trabajo citado constata, además, que la evolución de las importaciones está fuertemente correlacionada con el comportamiento de la demanda de exportaciones y de inversión y, por tanto, son los cambios en la composición de la demanda agregada los que determinan la senda de progreso de las importaciones. A este respecto, y aunque se extienda más allá del periodo temporal que aborda este trabajo, el repunte de las importaciones que ha acompañado a la recuperación de la economía española en 2014 y 2015, no puede desvincularse del fuerte crecimiento experimentado por la inversión en equipo y las exportaciones ${ }^{8}$.

En cuanto a la demanda de consumo, destaca la muy reducida dependencia importadora del consumo público, limitada, prácticamente, al aprovisionamiento de inputs.

\footnotetext{
${ }^{8}$ De acuerdo con la información suministrada por el Banco de España en su último informe anual, la inversión en bienes de equipo creció a tasas anuales superiores al 10\% en ambos años; las exportaciones por encima del 5\%. Son las rúbricas de la demanda final que han mostrado un mayor dinamismo (Banco de España, 2016).
} 
En el consumo privado no se observa que la intensa caída acaecida en la demanda con motivo de la crisis, se haya traducido en una menor dependencia externa. Por el contrario, en 2011, el peso en la demanda de las importaciones finales de bienes de consumo fue mayor que en 2008, y el contenido importado muy similar. No obstante, trabajos que incorporan información más reciente apuntan la existencia de un proceso de sustitución de importaciones de bienes de consumo, particularmente de bienes de consumo duradero, que ayuda a explicar el ajuste en las importaciones tras el colapso del comercio en 2009 (BBVA Research, 2014). Este proceso de sustitución de importaciones, que habría empezado a mostrarse tímidamente a partir de 2012, es explicado por la mejora competitiva de las producciones españolas derivada del proceso de ajuste y devaluación interna que, junto a una creciente sensibilidad de los consumidores españoles a los precios, parece haber inclinado las preferencias de los consumidores a favor de los productos nacionales (Banco de España, 2016). De ser así y afianzarse en el tiempo, este efecto tendería a contrarrestar el impulso sobre las importaciones derivado del crecimiento de la demanda de los componentes más dependientes de importaciones: las exportaciones y la inversión.

Figura 1

Dependencia externa de la demanda final nacional en la Unión Europa, EEUU y Japón, 1995 y 2011

(Porcentajes)

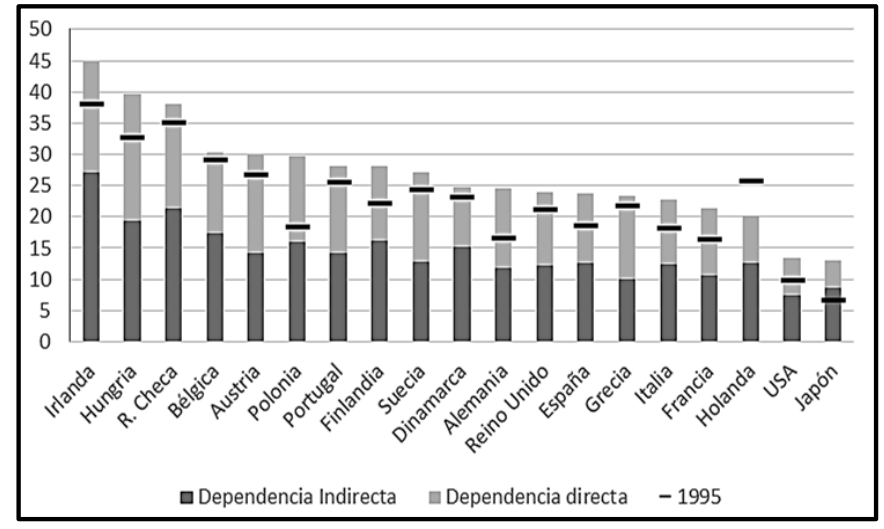

Fuente: Elaboración propia partir de ICIO.

Con objeto de determinar si el nivel que alcanza la dependencia importadora de la economía española difiere del de otras economías desarrolladas, en la Figura 1 se muestra la dependencia importadora de la demanda interna para los socios de la Unión Europea (UE) anteriores a la ampliación hacia el Este, los tres mayores países del grupo de Visegrad (Polonia, Hungría y República Checa), Estados Unidos y Japón. La dependencia externa para el año 2011 se 
presenta desagregada en sus dos componentes: dependencia directa e indirecta. Para observar los cambios registrados desde 1995 se ha incorporado también el grado de dependencia externa total en dicho año.

Puede observarse la distancia existente entre las economías seleccionadas. Mientras que en 2011, en Irlanda, la producción foránea abastece casi la mitad de la demanda final nacional, el 45\%; en Japón dicho porcentaje se reduce al $13 \%$. Las economías que muestran una mayor dependencia externa son las de dimensión más reducida (Irlanda, Bélgica y Austria) y las economías del Este (Hungría, República Checa y Polonia). En línea con lo esperado, dada la intensa integración comercial europea, las economías de la UE muestran un mayor grado de dependencia externa que Estados Unidos y Japón.

A priori, la dependencia externa de una economía depende del tamaño de los países, es decir, de sus posibilidades de acceder en el mercado nacional a inputs intermedios y bienes y servicios finales en la calidad y variedad requerida, de la competitividad-precio de los productos importados y de la composición de la demanda. Así, en los países de la UE de mayor dimensión y mercado (Alemania, Reino Unido, Francia e Italia), los requerimientos de importaciones para satisfacer la demanda nacional son relativamente más reducidos que en el resto de los socios.

Pero también, como ha puesto de manifiesto Martínez-Martín (2016) en su estimación de la demanda de importaciones para un conjunto de 27 países, la demanda de importaciones depende de otros factores que habitualmente no son considerados, entre los que destaca la participación en cadenas globales de valor. Ello explica la alta dependencia importadora de las economías más implicadas en redes trasnacionales de producción fundamentalmente como transformadoras de inputs foráneos (las economías del Este e Irlanda). Estas son, además, junto con Alemania, las economías que han experimentado los mayores aumentos en su dependencia de importaciones, en un contexto de generalizado crecimiento en las necesidades de importación del que solo se excluye Holanda. La internacionalización y reorganización de los procesos productivos que ha tenido lugar en Europa desde mediados de los noventa, en gran medida liderado por la economía alemana, ha supuesto una progresiva integración productiva en la que se combina el aprovechamiento de las ventajas en costes de los países del Este con las ventajas tecnológicas y en conocimiento propias de las economías más avanzadas (Marin, 2006; Breda, 2009; Díaz-Mora y García, 2016).

En cuanto al grado de dependencia relativo de la economía española, la mayor parte de los países europeos presentan, en 2011, un mayor grado de dependencia exterior que la economía española. Únicamente Francia, Italia, Grecia y Holanda, en Europa, presentan una demanda final nacional menos dependiente de las importaciones. A estos países se les añaden Estados Unidos y Japón. 
Merece la pena destacar el cambio que ha tenido lugar a lo largo del periodo estudiado en la situación relativa española respecto a Alemania. En 1995 la economía española mostraba un grado de dependencia frente al exterior claramente superior al de Alemania que, por otra parte, junto con Francia, Estados Unidos y Japón poseía los niveles más reducidos. El fuerte incremento en el contenido importado de las producciones alemanas vinculado a la internacionalización de sus procesos productivos (pasó del 7\% en 1995, al 12\% en 2011) ha invertido la situación.

Cuando la comparación se limita exclusivamente a la dependencia indirecta (Figura 1), aunque la economía española también se sitúa en una posición intermedia en el conjunto de los países objeto de estudio, su dependencia de inputs intermedios supera claramente a la de las economías centrales en la UE (Alemania, Francia, Reino Unido, y en menor grado a Italia). Un resultado en línea con los obtenidos para el año 2007 por Cabrero y Tiana (2012), que perdura desde 1995 y se mantiene para los distintos componentes de la demanda nacional, así como para las exportaciones (OCDE, 2015a).

Así pues, se puede concluir que aunque en el contexto de las economías europeas la dependencia importadora de la economía española no destaca especialmente; sin embargo, su tejido productivo es más dependiente de inputs intermedios importados que el de los países centrales de la UE. El análisis sectorial que se lleva a cabo en el siguiente epígrafe permitirá una detectar en qué producciones se concentra esta dependencia del exterior.

\section{ANÁLISIS SECTORIAL}

En la Tabla 2 se muestra el contenido importado de la producción española desagregada sectorialmente para los años 1995 y 2011. Con objeto de facilitar su comparación en el ámbito de la UE se han incluido, para 2011, dos medidas del contenido importado alternativas: el promedio de los valores sectoriales correspondientes a los 16 países que constituyen nuestra muestra una vez excluido España y el contenido importado que resulta de considerar la importancia relativa de cada país en el agregado. Se han incorporado los datos correspondientes a Alemania, Francia, Italia y Reino Unido por el interés que resulta de la comparación con las economías mayores del área comunitaria.

Los resultados que se obtienen confirman la disparidad sectorial en cuanto a las necesidades intermedias de importaciones y, como es conocido, ponen de manifiesto que la mayor dependencia importadora se presenta en la manufacturas, por lo que de aquí en adelante nos centraremos en dichas actividades. Pero antes de ello, merece la pena destacar la muy reducida dependencia del sector agrario español, la menor de todos los países estudiados excepto Italia, que ilustra la existencia de una especialización basada en la dotación de recursos naturales. Asimismo, se puede observar la escasa dependencia relativa de importaciones 
intermedias en la producción de servicios, un rasgo general al conjunto de las economías respecto a las que, en general, España presenta menores valores.

Tabla 2

Contenido importado de la producción

(Porcentajes)

\begin{tabular}{|c|c|c|c|c|c|c|c|c|}
\hline & \multicolumn{2}{|c|}{ ESPAÑA } & \multirow{2}{*}{\begin{tabular}{|c|}
$\begin{array}{c}\text { UE } \\
\text { (promedio) }\end{array}$ \\
2011 \\
\end{tabular}} & \multirow{2}{*}{$\begin{array}{c}\text { UE } \\
\text { (ponderado) } \\
2011\end{array}$} & \multirow{2}{*}{\begin{tabular}{|c|} 
ALEMANIA \\
2011
\end{tabular}} & \multirow{2}{*}{\begin{tabular}{|c|} 
FRANCIA \\
2011
\end{tabular}} & \multirow{2}{*}{\begin{tabular}{|c|} 
ITALIA \\
2011
\end{tabular}} & \multirow{2}{*}{$\begin{array}{c}\text { REINO } \\
\text { UNIDO } \\
2011\end{array}$} \\
\hline & 1995 & 2011 & & & & & & \\
\hline Agricultura & 9,2 & 18,9 & 25,0 & 22,8 & 22,6 & 20,0 & 16,1 & 24,0 \\
\hline Minería & 13,9 & 24,6 & 23,4 & 17,9 & 23,5 & 17,4 & 16,5 & 16,6 \\
\hline Alimentación, bebidas y tabaco & 17,6 & 27,4 & 29,6 & 28,1 & 27,5 & 22,7 & 23,1 & 26,3 \\
\hline $\begin{array}{l}\text { Textil, confección, cuero y } \\
\text { calzado }\end{array}$ & 21,0 & 33,3 & 33,5 & 30,2 & 30,7 & 31,4 & 27,5 & 26,8 \\
\hline Madera y corcho & 16,7 & 25,9 & 29,4 & 27,1 & 24,3 & 22,0 & 22,1 & 29,3 \\
\hline Papel y edición & 17,5 & 20,6 & 27,5 & 24,4 & 20,2 & 21,5 & 22,5 & 19,1 \\
\hline Coque y refinerias & 41,8 & 76,8 & 66,5 & 63,5 & 63,7 & 63,6 & 74,3 & 56,1 \\
\hline Química & 22,0 & 34,5 & 36,0 & 33,7 & 29,6 & 32,1 & 34,6 & 32,3 \\
\hline Caucho y plástico & 24,6 & 31,0 & 35,4 & 33,0 & 29,6 & 30,3 & 31,9 & 31,9 \\
\hline Minerales no metálicos & 18,5 & 26,2 & 31,2 & 28,0 & 23,9 & 22,8 & 26,7 & 29,1 \\
\hline Metalurgia & 23,2 & 34,0 & 45,8 & 45,0 & 45,2 & 29,0 & 47,5 & 51,7 \\
\hline Productos metálicos & 22,4 & 30,2 & 33,6 & 29,0 & 24,9 & 26,4 & 25,1 & 28,3 \\
\hline Maquinaria y equipo & 22,5 & 27,4 & 33,7 & 29,5 & 26,7 & 28,3 & 25,8 & 33,1 \\
\hline $\begin{array}{l}\text { Maq. de oficina, electrónica e } \\
\text { inst. precisión }\end{array}$ & 28,1 & 29,1 & 38,7 & 36,8 & 24,5 & 29,2 & 26,4 & 30,8 \\
\hline Maquinaria eléctrica & 24,7 & 35,9 & 38,1 & 33,1 & 25,8 & 32,2 & 30,2 & 35,4 \\
\hline Vehículos de motor & 36,5 & 46,1 & 43,5 & 38,6 & 31,4 & 37,6 & 33,9 & 44,4 \\
\hline Otros equipos de transporte & 24,1 & 33,3 & 36,1 & 37,8 & 35,4 & 43,2 & 29,5 & 35,3 \\
\hline Otras manufacturas & 20,1 & 27,7 & 33,5 & 30,4 & 26,3 & 28,9 & 24,2 & 28,1 \\
\hline Electricidad gas y agua & 18,0 & 33,1 & 29,7 & 25,5 & 24,1 & 23,6 & 35,9 & 37,6 \\
\hline Construcción & 15,6 & 15,6 & 25,6 & 22,2 & 18,3 & 16,8 & 16,8 & 16,8 \\
\hline Comercio y reparación & 8,8 & 11,5 & 16,1 & 14,3 & 11,6 & 10,1 & 15,0 & 12,4 \\
\hline Hoteles y restaurantes & 9,5 & 12,3 & 18,7 & 15,7 & 13,6 & 13,9 & 14,5 & 16,3 \\
\hline Transporte & 10,8 & 19,6 & 23,6 & 22,3 & 19,4 & 14,2 & 15,5 & 15,2 \\
\hline Correo y telecomunicaciones & 5,2 & 16,4 & 16,4 & 16,0 & 16,1 & 12,5 & 11,9 & 15,5 \\
\hline Intermediación financiera & 5,0 & 7,2 & 11,1 & 12,6 & 10,2 & 6,3 & 5,4 & 9,3 \\
\hline Actividades inmobiliarias & 3,9 & 4,0 & 9,4 & 5,2 & 3,2 & 2,4 & 1,7 & 4,2 \\
\hline Alquiler de maquinaria y equipo & 8,7 & 16,1 & 16,2 & 20,4 & 4,6 & 9,7 & 16,5 & 12,0 \\
\hline Informática & 8,0 & 13,4 & 16,5 & 25,3 & 10,4 & 7,6 & 11,7 & 10,5 \\
\hline $\begin{array}{l}\text { I+D y otras actividades } \\
\text { empresariales }\end{array}$ & 8,8 & 12,3 & 15,7 & 12,8 & 9,0 & 9,3 & 11,2 & 11,5 \\
\hline Administración pública & 6,0 & 8,1 & 9,7 & 10,1 & 8,9 & 8,6 & 5,4 & 11,1 \\
\hline Educación & 2,5 & 4,3 & 6,0 & 6,0 & 4,3 & 4,2 & 2,9 & 6,7 \\
\hline Servicios sociales y salud & 9,4 & 12,2 & 13,0 & 10,1 & 8,2 & 6,9 & 10,6 & 13,9 \\
\hline Otros servicios & 7,3 & 12,3 & 16,2 & 13,3 & 8,8 & 12,1 & 13,4 & 11,2 \\
\hline Manufacturas & 23,2 & 35,4 & 38,2 & 37,5 & 30,3 & 32,1 & 31,4 & 33,3 \\
\hline Alta y medio alta tecnología & 28,0 & 37,0 & 38,7 & 34,8 & 28,9 & 34,5 & 29,6 & 35,4 \\
\hline Medio baja tecnología & 22,0 & 30,8 & 37,3 & 35,7 & 32,0 & 27,3 & 32,1 & 34,5 \\
\hline Baja tecnología & 18,4 & 26,8 & 29,9 & 27,7 & 25,4 & 23,8 & 24,4 & 24,7 \\
\hline
\end{tabular}

Fuente: Elaboración propia partir de ICIO.

Como se ha señalado, el mayor contenido importado se presenta en las manufacturas, alcanzándose un valor del 35,4\% en el año 2011 que significa que 
más de un tercio de la producción manufacturera española se genera fuera de nuestras fronteras. Esta cuantía, que supera en 12 puntos porcentuales su valor en 1995, es menor que la que muestran los indicadores correspondientes a la UE; incluso cuando se pondera por el peso de los países y el contenido importado en la UE se reduce, dada la menor dependencia importadora de los países grandes. Sin embargo, supera claramente la dependencia importadora de las grandes economías europeas, así como la de algunas economías de pequeña dimensión como Holanda o Dinamarca que no se recogen en el cuadro. Así pues parece que en el contexto de los grandes países europeos, la dependencia importadora de las manufacturas españolas es relativamente elevada, si bien, claramente inferior a la de los países fuertemente especializados en la transformación de inputs importados (economías del Este) y algunas de las economías de menor dimensión (Irlanda, Bélgica o Portugal).

Este resultado, además, es aplicable a la mayoría de las ramas de actividad, de modo que el contenido importado de las producciones españolas excede al de las economías más avanzadas prácticamente en todas las manufacturas ${ }^{9}$. Es decir, la dependencia externa de la producción industrial española no es consecuencia de que su patrón productivo se escore hacia las producciones más dependientes del exterior, sino un rasgo que caracteriza al conjunto de la actividad industrial y que ya evidenciaron Cabrero y Tiana (2012) para el año 2007, antes del inicio de la crisis.

Para facilitar el análisis sectorial se han desagregado las manufacturas según su intensidad tecnológica en tres grupos: alta y media alta intensidad tecnológica, media-baja y baja ${ }^{10}$. El contenido importado está relacionado con la intensidad tecnológica de las manufacturas, siendo las industrias más vinculadas a los desarrollos tecnológicos las que detentan las mayores necesidades de importaciones y donde mayores son las diferencias con respecto a las economías centrales de la UE, particularmente Alemania e Italia.

El elevado contenido importado de las industrias de alto contenido tecnológico es provocado fundamentalmente, por la altísima dependencia de

${ }^{9}$ La excepción más significativa es metalurgia, donde el contenido importado está fuertemente vinculado a la dotación de recursos. También se observa un menor contenido importado en "Otro material de transporte" que podría estar relacionado con la diferente composición de un sector que engloba producciones tan misceláneas.

${ }^{10}$ Las manufacturas incluidas en alta y media-alta intensidad tecnológica son química, maquinaria y equipo, maquinaria de oficina, electrónica e instrumentos de precisión, maquinaria eléctrica, vehículos de motor y otros equipos de transporte. En media-baja tecnología están comprendidas las ramas de caucho y plástico, minerales no metálicos, metalurgia y productos metálicos. Aunque para el total de manufacturas se considera la producción de coque y refinerías, esta se ha excluido del subagregado para evitar la distorsión que implica la muy elevada dependencia del petróleo en la economía española. Por último, en baja tecnología se consideran alimentación, bebidas y tabaco, textil, confección cuero y calzado, papel y edición, madera y corcho y otras manufacturas. 
inputs foráneos en la fabricación de vehículos de motor, un 46\% en 2011, expresión de cómo participan las empresas situadas en España en el proceso de especialización vertical que caracteriza a este sector productivo. La relevancia que alcanza nuestro país en la producción y exportación de automóviles, con la presencia de plantas de montaje de las principales marcas de automoción europeas (Volkswagen o Renault), americanas (Ford), o japonesas (Nissan), explica la intensidad de la dependencia de inputs intermedios importados. Aunque la industria española ha desarrollado una importante industria auxiliar, su especialización en el ensamblaje final le diferencia de otras economías, como Italia o Alemania, más orientadas hacia la provisión de partes y componentes para su transformación y montaje en países con ventajas de coste que, en consecuencia, son menos dependientes de los suministros exteriores (Blázquez et al., 2010).

Esta explicación, que vincula la dependencia tecnológica a la participación en cadenas globales de valor lideradas por empresas de capital extranjero, también parece adecuada para explicar el alto contenido importado relativo de otras dos ramas de intensidad tecnológica media-alta: química y maquinaria eléctrica. En la primera de ellas destaca, además, la alteración que se ha producido en el periodo estudiado. En 1995, solo tres economías, Alemania, Francia y Polonia, mostraban un contenido importado inferior al de la química española; sin embargo, el intenso aumento en las necesidades de importación ha colocado a la química española en 2011 entre las más dependientes del exterior en el entorno europeo. Aunque esta creciente necesidad de inputs puede deberse a un cambio en la composición de la producción hacia las producciones con mayores requerimientos de inputs importados, el dinamismo exportador e importador del sector, especialmente de los productos farmacéuticos, que García y Tello (2011) justifican por la mejora competitiva asociada a la presencia de grandes multinacionales en el sector, parece avalar la relación entre capital extranjero, especialización vertical y dependencia importadora en los sectores más intensivos en tecnología.

Esta asociación entre dependencia importadora y especialización vertical liderada por el capital extranjero encuentra sustento teórico en el modelo planteado por Antras (2003), que demuestra que la forma en que las empresas organizan la internacionalización de los procesos productivos y se abastecen de inputs exteriores (a través del mercado o mediante comercio intrafirma) no es independiente de la intensidad factorial de los inputs requeridos. En los sectores relativamente más intensivos en capital, tecnología y conocimientos, las empresas tienden a integrar verticalmente sus procesos de producción internacionalizados porque de ese modo pueden controlar mejor su calidad, requisitos y necesidades de inversión. Su contrastación empírica para la economía estadounidense confirma las predicciones del modelo: en las ramas con mayores exigencias tecnológicas tienen una mayor presencia las importaciones de inputs intrafirma. 
Fariñas y Martín (2011) encuentran los mismos resultados para las manufacturas españolas.

Más aún, Álvarez y Myro (2016) han puesto de relieve que en la economía española la relación entre capital extranjero y dependencia de inputs intermedios no se limita a las industrias tecnológicamente más avanzadas. Su estimación de la intensidad importadora en relación a las ventas elaborada a partir de los datos de la Central de Balances del Banco de España muestra que las filiales del capital extranjero poseen una mayor orientación hacia el abastecimiento externo de inputs que las empresas nacionales. Esta mayor dependencia de importaciones, además, es un rasgo generalizado del tejido productivo español que incrementa en 6 puntos porcentuales la propensión media a importar de las empresas españolas.

Entre las industrias de baja tecnología destaca la elevada dependencia relativa de inputs intermedios del exterior de la rama textil, confección, cuero y calzado (33,3\% en 2011) que no solo supera a las restantes ramas del grupo sino que también es mayor que la dependencia foránea de estas actividades en Alemania, Francia o Italia. Para valorar este hecho adecuadamente hay que tener en cuenta que el fuerte aumento en las necesidades de importaciones intermedias de la industria textil española se ha acompañado de un gran dinamismo exportador que ha permitido incrementar su cuota exportadora mundial en la primera década de este siglo ${ }^{11}$. En cambio, gran parte de las economías avanzadas, como Francia e Italia, veían reducir su participación en las exportaciones mundiales incapaces de hacer frente a la intensa competencia procedente de países de bajo coste. Esto parecería indicar que la industria textil y de confección ha desarrollado estrategias empresariales basadas en la diferenciación de producto y en la fragmentación internacional de los procesos productivos, con un creciente abastecimiento procedente de países con ventajas de costes que han posibilitado la mejora competitiva que explica sus buenos resultados en los mercados mundiales (García y Tello, 2011).

Así pues, el elevado contenido importado de las producciones españolas parece responder a un proceso de especialización vertical que se adecúa a las ventajas propias de la economía y tiene efectos relevantes sobre su posición competitiva $^{12}$. La Figura 2 muestra, para las diferentes ramas, la relación entre

${ }^{11}$ Este dinamismo exportador se limita a las ramas de textil y confección, porque la industria del cuero y calzado ha reducido desde comienzos de siglo su participación en la producción y exportaciones.

12 En esta línea, Díaz-Mora et al. (2007) muestran que el fuerte incremento en los aprovisionamientos de inputs materiales del exterior de las manufacturas españolas responde a la extensión de estrategias de internacionalización que implican una fragmentación de los procesos productivos. Fuster y Martínez-Mora (2013) constatan un aumento en la dependencia de servicios intermedios importados de las manufacturas españolas que, sin embargo, parece estar más relacionado con la sustitución de proveedores nacionales por extranjeros. 
la variación en el valor añadido del exterior incorporado en la producción nacional y las rentas generadas en el país. La elevada correlación que se percibe pone de manifiesto que el aprovisionamiento externo de inputs ejerce un efecto impulsor sobre las rentas nacionales consecuencia de su positiva incidencia sobre la competitividad ${ }^{13}$.

Figura 2

Evolución del valor añadido exterior y doméstico contenido en la demanda final y las exportaciones (Tasas de variación anuales acumulativas, 1995-2001)

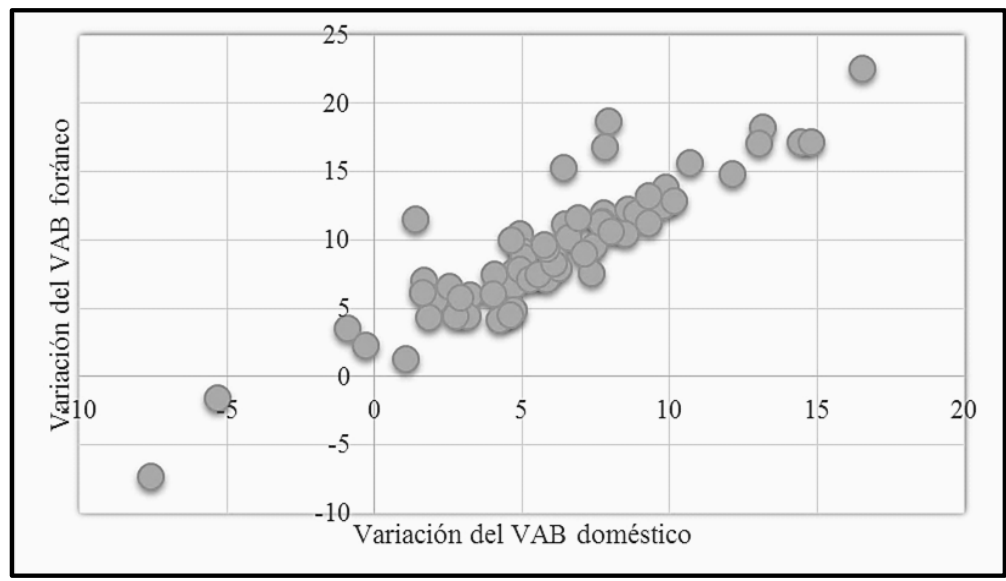

Fuente: Elaboración propia partir de ICIO.

Las importaciones intermedias se configuran como un elemento clave de la competitividad. A través de ellas las empresas se benefician de la transmisión de las tecnologías más avanzadas y consiguen inputs de mejor calidad y precio. La especialización resultante conlleva un uso de los recursos productivos más eficiente y, en consecuencia, un impulso a la mejora productiva. Para la industria española, Fariñas (2014) encuentra que el abastecimiento de inputs en los mercados internacionales, frecuente entre las grandes empresas, tiene una presencia limitada entre las empresas de menor dimensión, a pesar del efecto positivo que la decisión de aprovisionamiento exterior de inputs tiene sobre la productividad de las empresas. En definitiva, en una economía globalizada que

${ }^{13}$ La positiva relación entre abastecimiento externo de inputs y la generación de rentas en el país también ha sido puesta de manifiesto por Kummritz (2015) para las exportaciones. Para una muestra de 50 economías con distinto grado de desarrollo y 20 sectores de actividad, este autor constata que la incorporación de valor añadido del exterior en las exportaciones tiene un efecto impulsor sobre las rentas nacionales vinculadas a la exportación en los países de renta media y alta (no así en los de menor nivel de renta, cuya capacidad de absorción es más limitada). Sus resultados apuntan que el valor añadido procedente del exterior que se incorpora en las exportaciones es complementario del valor añadido doméstico. 
participe activamente de la división internacional del trabajo y desee beneficiarse de las ventajas de la especialización no parece factible, ni deseable, plantearse un menor contenido importado de la producción.

Sin embargo, el análisis sectorial de la dependencia importadora también revela algunas de las debilidades del patrón industrial español. La dependencia importadora directa en las industrias de mayor contenido tecnológico es muy elevada (Figura 3). La producción foránea abastece más de la mitad del consumo privado de bienes de alta y media tecnológica; el 60 por ciento en el caso de los bienes de inversión. Un porcentaje sustancialmente superior al de las economías centrales europeas (muy particularmente al de Alemania, pero también al de Francia o Italia).

Figura 3

Dependencia directa en las industrias de alta y media tecnología, 2011

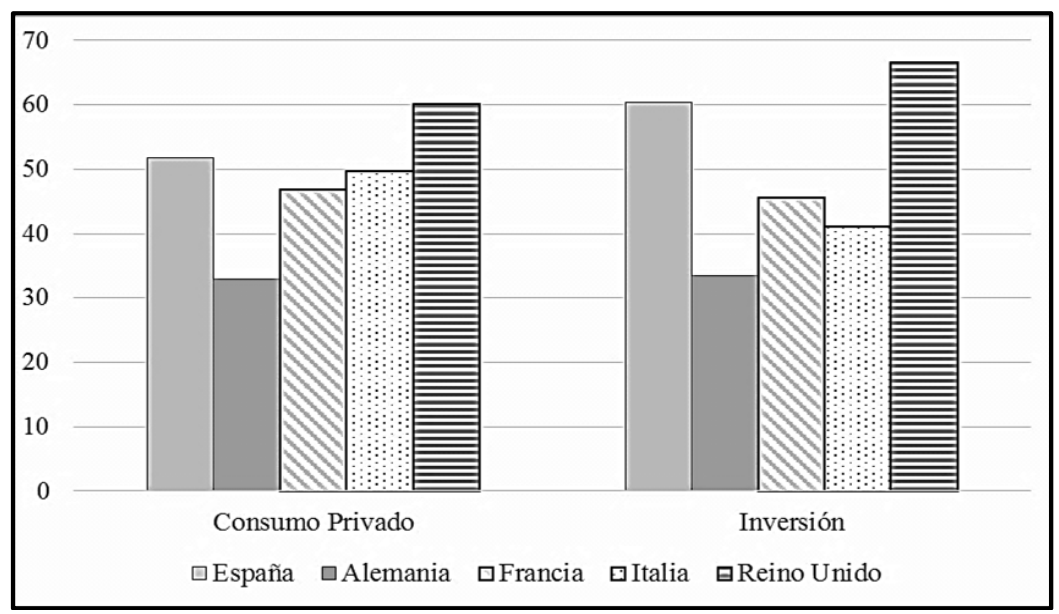

Fuente: Elaboración propia partir de ICIO.

La dependencia externa del consumo y la inversión es extraordinariamente alta en maquinaria de oficina, electrónica e instrumentos de precisión donde la competitividad requiere un mayor esfuerzo en innovación y desarrollo. El insuficiente desarrollo de este tipo de producciones en nuestro país, que se traduce en que España sea de las economías europeas con una menor presencia en el patrón industrial de las manufacturas con mayor contenido tecnológico, es la expresión de la dependencia tecnológica que sigue caracterizando al tejido productivo. Solo a través de una mejora en el potencial tecnológico e innovador de la economía española se podrá avanzar en el desarrollo de estas producciones que, por otra parte son las que muestran un mayor dinamismo en los mercados mundiales, y aminorar su dependencia importadora. 


\section{CONCLUSIONES}

La estimación de la dependencia externa de la economía española que se ha realizado en este trabajo a partir de las Tablas Input-Output Internacionales (ICIO) ha puesto de manifiesto una tendencia creciente desde mediados de los años noventa, que tras quebrarse en 2009 con el colapso del comercio mundial, ya había recuperado sus valores pre-crisis en 2011. La mayor dependencia que muestran los componentes de la demanda más dinámicos en la recuperación, exportaciones e inversión, sugiere la continuidad de esta senda evolutiva que, por otra parte, es común a la que han experimentado el resto de las economías europeas conforme avanzaba la integración de la economía mundial.

En el contexto europeo, el grado de dependencia de nuestra economía no parece especialmente elevado teniendo en cuenta tanto su tamaño relativo como su participación en el proceso de reordenación geográfica de los procesos productivos que ha tenido lugar en las últimas décadas. Sin embargo, no deja de ser preocupante la mayor dependencia importadora respecto a los países centrales de la Unión Europea, puesto que reduce las posibilidades de crecimiento de la producción y la renta ante un mismo ascenso de la demanda y, en consecuencia, exige un mayor impulso de la demanda interna y, sobre todo de las exportaciones, para avanzar en el proceso de convergencia en renta per cápita.

El análisis sectorial revela claramente que, de forma generalizada, las producciones industriales son más dependientes de inputs exteriores que las de Alemania, Francia, Italia y, en menor medida, el Reino Unido. Ello parece ser la consecuencia de un proceso de especialización en el que sobre todo en las industrias más intensivas en tecnología, las empresas españolas que participan en cadenas globales de valor, lideradas por capital extranjero, llevan a cabo las fases más vinculadas a la transformación y el ensamblaje de inputs. Pero también, es el resultado de la creciente adopción de estrategias de aprovisionamiento exterior de inputs con el objetivo de mejorar la eficiencia productiva. Así, la dependencia de inputs exteriores ha de ser interpretada como una vía de mejora de la competitividad industrial.

Sin embargo, la intensa dependencia de la demanda de importaciones finales, particularmente en las industrias con mayores requerimientos tecnológicos, expresa el deficiente desarrollo en nuestro país de las producciones industriales con mayor potencial de crecimiento. Un rasgo que distingue nuestro patrón industrial del de otras economías más adelantadas y pone énfasis en la necesidad de avanzar en el esfuerzo investigador e innovador para mitigar la dependencia importadora. 


\section{REFERENCIAS BIBLIOGRÁFICAS}

ALVÁREZ, E. y MYRO, R. (2016). "Situación comparada de la industria española". En R. Myro (dir.): Una nueva política industrial para España (pp. 17-49). Consejo Económico y Social.

AMADOR, J.; CAPPARIELLO, R. y STEHRER, R. (2015). "Global value Chains: A view from the Euro Area". Asian Economic Journal, vol. 29, n² 2, pp.99-120.

ANTRAS, P. (2003). "Firms, Contracts and Trade Structure". Quarterly Journal of Economics, 118, pp. 1375-1418.

BALDWIN, R. y LÓPEZ-GONZÁLEZ, J. (2015). "Supply-chain Trade: A Portrait of Global Patterns and Several Testable Hypotheses". The World Economy, vol. 38 (11), pp.1682-1721.

BANCO DE ESPAÑA. (2016). Informe anual 2015. Madrid.

BBVA RESEARCH. (2014). "Made in Spain: ¿está cambiando la preferencia de los consumidores españoles por los productos nativos?". Situación Consumo, Primer Semestre, pp. 21-29.

BLAZQUEZ, L., DÍAZ-MORA, C. y GANDOY, R. (2010). "Tendencias en la localización de la industria europea de automoción: Un análisis a partir del comercio de partes y componentes". Economía Industrial, $n^{\circ} 376$, pp.75-95.

BREDA, E., CAPPARIELLO, R., y ZIZZA, R. (2009). "Vertical specialisation in Europe: evidence from the import content of exports". En Firms' Objectives and Internal Organisation in a Global Economy (pp. 189-212.). Palgrave Macmillan UK.

BUSSIĖRE, M. C. (2013). "Estimating trade elasticities: Demand elasticities and the trade collapse of 2008-09". American Economic Journal: Macroeconomics 5 (3), pp. 118-151.

CABRERO, A. y TIANA, M. (2012). "Contenido importador de las ramas de actividad en España". Boletin Económico del Banco de España, febrero, pp.45-68.

CONSTANTINESCU, C. A. (2015). "The Global Trade Slowdown. Cyclical or Structural?" World Bank Policy Research. Working paper, $\mathrm{n}^{\circ} 7158$.

DE BACKER, K. y MIROUDOT, S. (2013). Mapping Global Value Chains. OECD Trade Policy Papers 159, OECD Publishing.

DIAZ-MORA, C. y GARCÍA, E. (2016). "Centre-periphery integration: Building European production chains". Spanish Economic and Financial Outlook, vol. 5, no 4, pp. 79-94.

DIAZ-MORA, C. y GANDOY, R. y GONZÁLEZ, B. (2007). "Fragmentación internacional de la producción en las manufacturas españolas". Papeles de Economía Española, $\mathrm{n}^{\circ} 112$, pp. 74-88.

ESCAITH, H. L. (2010). International Supply Chains and Trade Elasticity in Times of Global Crisis. Economic Research and Statistic Division Staff Working paper ERSD2010-08, Geneva: World Trade Organization.

ESCAITH, H. L. (2014). Exploring the Policy Dimension of Trade in Value-Added. MPRA Paper $n^{\circ} 59891$, University Library of Munich.

EUROPEAN CENTRAL BANK. (2015). "Understanding the Weakness in World Trade". Economic Bulletin 3, pp.33-42.

FARIÑAS, J. C. (2014)."El offshoring: rasgos y efectos sobre la productividad empresarial". En Fariñas y Fernández de Guevara, J. (dir.) "La empresa española ante la crisis del 
modelo productivo" (pp. 55-98). Fundación BBVA.

FARIÑAS, J.L. y MARTÍN, A. (2011). "Organización de la producción, comercio exterior y productividad". Información Comercial Española, nº 82, pp. 217-243.

FUSTER, B. y MARTÍNEZ-MORA, C. (2013). "Offshoring of Services in Spain: International fragmentation of Activities or Change in Procurement Sources?" Estudios de Economía Aplicada, vol. 31(2), pp.655-676.

GARCÍA, C. y TELLO, O. (2011). "La evolución de la cuota de exportación de los productos españoles en la última década: el papel de la especialización comercial y de la competitividad". Boletín Económico de Banco de España, Mayo, pp. 49-60.

HOEKMAN, B. (2015). The Global trade slowdown: A new normal? VoxEU.org eBook.

HUMMELS, D., ISHII, J. y YI, K. . (2001). "The Nature and Growth of Vertical Specialization in World Trade". Journal of International Economics 54, pp. 75-96.

JONES, L. W. (2014). The Similarities and Differences among Three Major Inter-Country Input-Output Database and their Implications for trade in Value-Added Estimates. U.S. International Trade Commission, Working Paper $n^{\circ}$ 2014-12B.

KOOPMAN, R., WANG, Z. and WEI, S-J. (2014). "Tracing Value Added and Double Counting in Gross Exports" . American Economic Review 104(2), pp 459-494.

KOWALSKI, P., LÓPEZ GONZÁlEZ, J., RAGOUSSIS, A. y UGARTE, C. (2015). Participation of Developing Countries in Global Value Chains: Implications for Trade and Trade-Related Policies. OECD Trade Policy Papers $n^{\circ} 179$, OECD Publishing.

KUMMRITZ, V. (2015). Global Value Chains: Benefiting the Domestic Economy? Graduate Institute of International and Development Studies, International Economics Department Working Paper Series, IHEIDWP02-2015.

MARIN, D. (2006). "A New International Division of Labor in Eastern Europe:Outsourcing and Offshoring to Eastern Europe". Journal of the European Economic Association n०4, vol.2(3), pp. 612-622.

MARTINEZ-MARTíN, J. (2016). Breaking Down World Trade Elasticities: A Panel ECM Approach Banco de España, Documento de trabajo nº 1614

MUÑOZ CIDAD, C. (2000). Las cuentas de la nación. Introducción a la Economía Aplicada. Civitas. Madrid

OCDE. (2011). "Import content of export". En OECD Science, technology and Industry Scoreboard (pp. 178-179). OECD Publishing.

OCDE. (2015a). Trade in value added: Spain.

OCDE. (2015b). TiVA 2015 indicators-definitions. Obtenido de https://www.oecd.org/sti/ ind/tiva/TIVA_2015_Indicators_Definitions.pdf

TIMMER M.P., DIETZENBACHER, E., LOS, B.,STHERER, R. y VRIES, G.J. (2015). "An Illustrated User Guide to the World Input-Output Database: the Case of Global Automotive Production". Review of International Economics, 23(3), pp.575-605.

TIMMER M.P., ERUMBAN, A.A.; LOS, B.,STHERER, R. y VRIES, G.J. (2014). "Slicing Up Global Value Chains" Journal of Economic Perspectives 28 (2), pp. 99-118. 